\title{
Primeira descrição de pseudoparasitismo por esporocistos de Monocystis sp. em tatu- galinha, Dasypus novemcinctus (Linnaeus, 1758) - relato de caso
}

\author{
[First description of pseudoparasitism by sporocysts of Monocystis sp. in nine banded armadillo \\ Dasypus novemcinctus (Linnaeus, 1758) - case report] \\ C.M. Prado ${ }^{1}$, A.P.M. Candeias $^{2}$, A.L.V. Beninca ${ }^{2}$, S. Wu ${ }^{2}$, R.J. Piccoli ${ }^{2}$, \\ L.Q.F.C. Borges ${ }^{4}$, A.L. Carvalho ${ }^{3}$, N.L.M. Fernandes ${ }^{3}$ \\ ${ }^{1}$ Programa de pós-graduação - Universidade Federal do Paraná - Curitiba, PR \\ ${ }^{2}$ Programa de pós-graduação - Universidade Federal do Paraná - Palotina, PR \\ ${ }^{3}$ Universidade Federal do Paraná - Palotina, PR \\ ${ }^{4}$ Secretaria de Meio Ambiente - Prefeitura Municipal de Toledo - Toledo, PR
}

C. M. Prado

https://orcid.org/0000-0003-0272-0709 A. P. M Candeias

https://orcid.org/0000-0001-8906-7962 A. L. V. Beninca

https://orcid.org/0000-0001-7078-7839 $\mathrm{S} . \mathrm{Wu}$

https://orcid.org/0000-0001-9934-5110 R. J. Piccoli

https://orcid.org/0000-0001-7741-8999

L. Q. F. C. Borges

https://orcid.org/0000-0002-6419-900X A. L. Carvalho https://orcid.org/0000-0003-1926-0497

N. L. M. Fernandes

https://orcid.org/0000-0002-9391-5765

O presente artigo é um relato sobre uma fêmea de D. novemcinctus que foi capturada de forma imprevista, durante um projeto de avaliação sanitária de animais silvestres sinantrópicos do município de Toledo/PR (autorização Sisbio/Ibama n. 55109-1). Durante a avaliação física, amostras de fezes foram coletadas após defecação espontânea e encaminhadas para análise. O material foi submetido aos métodos de flutuação simples em solução hipersaturada de cloreto de sódio e centrífugo-flutuação em sacarose. Na leitura das lâminas obtidas pelo método de centrífugo-flutuação em sacarose, foram observadas estruturas com comprimento de aproximadamente $10 \mu \mathrm{m}$, forma bicônica e um tampão em cada extremidade, compatíveis com esporocistos de Monocystis sp. Protozoários do filo Apicomplexa, os Monocystis sp., são geralmente parasitas de anelídeos, como as minhocas. Esse organismo é comumente correlato a pseudoparasitismo em vários animais, porém não foram encontrados relatos de pseudoparasitismo desse agente em tatus-galinhas, logo essa é a primeira descrição de Monocystis sp. em espécime de $D$. novemcinctus.

Palavras-chave: diagnóstico, protozoários, pseudoparasitas, animais silvestres

\begin{abstract}
The present article is an account of a female of $\mathrm{D}$. novemcinctus which was captured during a sanitary evaluation project of synanthropic wild animals of the municipality of Toledo/PR (license SISBIO/ICMBIO $n^{\circ}$. 55109-1). During a physical evaluation, samples of feces were collected, after spontaneous defecation, and sent for analysis. The material was subjected to simple flotation methods in hypersaturated sodium chloride solution and centrifugal-flotation solutions in sucrose. In a test reading

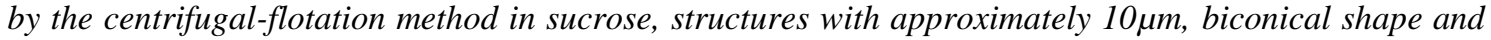
one plate at each end were observed, compatible with sporocysts of Monocystis sp. Protozoa of the phylum Apicomplexa, like Monocystis sp., it is common parasitic annelids, such as worms. This organism is a correlate of pseudoparasitism in several animals but was not found to describe this product as an agent in nine banded armadillos, so this is a first description of Monocystis sp. in specimen of D. novemcinctus.
\end{abstract}

Keywords: diagnosis, protozoa, pseudoparasites, wild animals

Recebido em 30 de outubro de 2018

Aceito em 24 de janeiro de 2019

E-mail: c.melchior.mv@gmail.com 


\section{INTRODUÇÃO}

O tatu-galinha (Dasypus novemcinctus) é um mamífero da ordem Cingulata e superordem Xenarthra, com hábito semifossorial, comportamento crepuscular e/ou noturno e ampla distribuição geográfica, que abrange desde o sul dos Estados Unidos até o Uruguai (Eisenberg e Redford, 1999; Vizcaíno e Loughry, 2008; Silva et al., 2015). Por se tratar de uma espécie relativamente tolerante a alterações ambientais, a espécie pode ser observada em diferentes ambientes, incluindo os urbanos (Silva et al., 2015). Esse fato proporcionou o aumento de seu contato com a população humana e com animais domésticos, o que possibilita a disseminação de agentes infecciosos e parasitários para novos hospedeiros e ambientes (Menezes, 2005). Dessa forma, o monitoramento sanitário desses indivíduos é importante para identificação de agentes de relevância à saúde pública, principalmente em localidades onde a espécie, por motivos culturais (ou não), faz parte da dieta de populações humanas (Antunes, 2007).

Em minhocas, protozoários do filo Apicomplexa, entre eles o Monocystis sp., afetam exclusivamente as vesículas seminais e promovem infertilidade (Field e Michiels, 2006, Bandyopadhyay et al., 2009; Velavan et al., 2010). Indivíduos do gênero Monocystis passam por três fases bem definidas durante seu ciclo de vida: (i) fase de infecção - em que minhocas se infectam pela ingestão de oocistos com esporozoítos no solo, que progridem para o sistema circulatório e invadem o lúmem da vesícula espermática onde amadurecem como trofozoítos e destroem os espermatócitos em desenvolvimento; (ii) fase sexual - em que dois ou mais gamontes se fundem e formam um gametócito (com um cisto envelopado), cujas divisões nucleares resultam na formação de um zigoto que secreta uma membrana ao redor do oocisto; (iii) fase de dispersão - em que a membrana do oocisto endurece, resultando em esporocistos em formato fusiforme típico, a partir da qual duas ou três divisões celulares seguem para formar oito esporozoítos dentro de um esporo. Nesse ponto, o gametócito se rompe, liberando os muitos esporocistos no fluido seminal e, eventualmente, no meio ambiente, para repetir o ciclo de vida (Field e Michiels, 2006; Velavan et al., 2010). Por meio de um ciclo errático, a minhoca, ao ser digerida por um vertebrado, libera os esporocistos, os quais saem junto com as fezes deste, que passa a ser um hospedeiro acidental (Field e Michiels, 2006).

O objetivo deste trabalho foi relatar a primeira descrição de caso de pseudoparasitismo por esporocistos de Monocystis sp. em tatu-galinha.

\section{CASUÍSTICA}

Durante projeto de pesquisa para avaliação sanitária de animais silvestres em remanescentes florestais do município de Toledo/PR (autorização Sisbio/ICMBIO n. 55109-1), realizou-se a captura imprevista de uma fêmea de tatu-galinha (Dasypus novemcinctus) nas coordenadas 24\%42'23”S 53\%43'37.54”O. O animal, com peso de $4,90 \mathrm{~kg}$, foi capturado em uma armadilha de arame galvanizado modelo Tomahawk (dimensões $1,15 \mathrm{~m}$ x $0,4 \mathrm{~m} \times 0,6 \mathrm{~m}$ ), iscada com banana, mamão e tilápia-do-nilo (Oreochromis niloticus). Apesar de se tratar de captura imprevista, realizou-se a contenção física para exame clínico, coleta de sangue pela veia caudal e coleta de fezes após defecação espontânea durante o procedimento de captura.

As amostras de fezes foram encaminhadas para análise, que ocorreu pelos métodos de flutuação simples em solução hipersaturada de cloreto de sódio e centrífugo-flutuação em sacarose, conforme protocolo estabelecido por Willis (1921) e Sheather (1923), respectivamente.

Na leitura das lâminas obtidas pelo método de centrífugo-flutuação em sacarose, foram observadas estruturas com comprimento de aproximadamente $10 \mu \mathrm{m}$, com forma bicônica e com um tampão em cada extremidade (Fig. 1), compatíveis com esporocistos de Monocystis sp. 


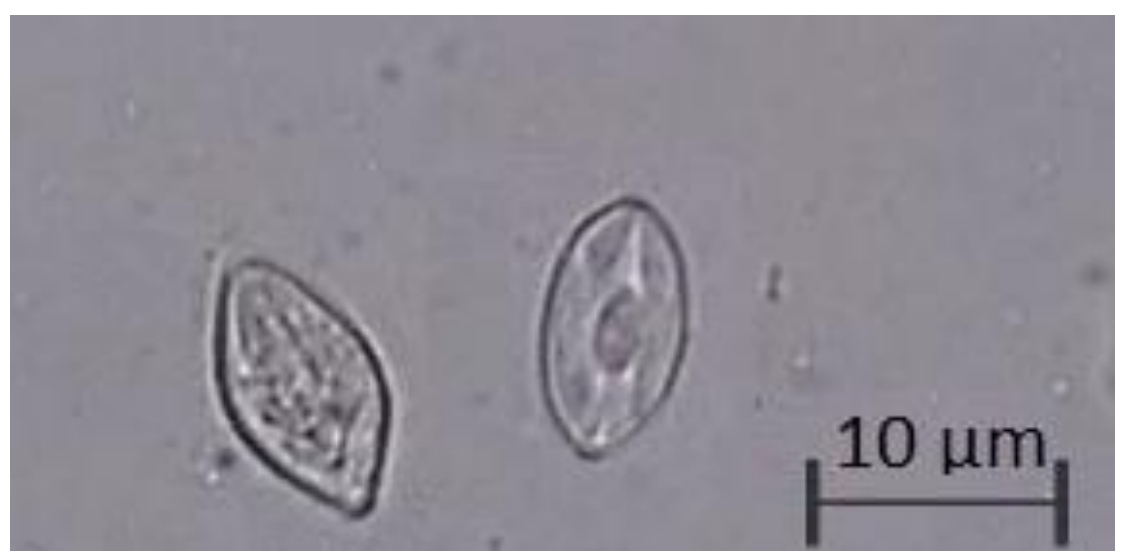

Figura 1. Esporocistos de Monocystis sp. encontrados em fezes de tatu-galinha (Dasypus novemcinctus (Linnaeus, 1758) por meio de método de centrífugo-flutuação em solução de sacarose. Esporocistos com comprimento de aproximadamente $10 \mu \mathrm{m}$. Aumento de 400x.

\section{DISCUSSÃO E CONCLUSÕES}

A ocorrência de pseudoparasitismo é relatada em diferentes espécies de animais, nas quais está normalmente associada aos hábitos alimentares desses indivíduos (Blagburn, 2010). Os tatus apresentam arcada com molares primitivos e pouco adaptados à mastigação de alimentos duros. Dessa maneira, um dos constituintes de sua dieta são pequenos invertebrados, como anelídeos, que possuem como representante mais comum as minhocas (Miranda, 2014; Silva et al., 2015).

A detecção de esporocistos de Monocystis sp. nas fezes desse exemplar de tatu-galinha reforça a importância do conhecimento sobre dieta dos animais e composição parasitária desses itens alimentares, principalmente quando se realizam análises de amostras de animais silvestres. Adicionalmente, o conhecimento sobre a morfologia e o ciclo de vida de pseudoparasitas permite evitar diagnósticos falso-positivos. Além disso, se forem consideradas populações de tatus em cativeiro, sobretudo aquelas com alta densidade de indivíduos, a prevalência desses esporozoítos nas fezes dos indivíduos pode levar ao entendimento equivocado da necessidade de tratamento desses animais.

Tal situação é favorecida pela escassez de literatura sobre agentes parasitários de animais silvestres, o que reforça a necessidade de descrição, por meios científicos, de novos achados nesses animais. Esta é a primeira descrição de Monocystis sp. em tatu-galinha. Embora o mesmo parasita já tenha sido relatado em outros animais, como cães, gatos e aves, vale ressaltar que não há comprovação de seu efeito patogênico sobre hospedeiros vertebrados.

\section{REFERÊNCIAS}

ANTUNES, J.M.A.P. Pesquisa de Mycobacterium leprae em tatus selvagens da espécie Dasypus novemcinctus no Estado do Espírito Santo. 2007. 105f. Dissertação (Mestrado em Doenças Infecciosas) Universidade Federal do Espírito Santo, Vitória, ES.

BANDYOPADHYAY, P.K.; MITRA, A.K.; GOÇMEN, B. Observation on Monocystis constricta n. sp. (Protozoa: Apicomplexa: Monocystidae) from na Indian Earthworm, Eutyphoeus quaripapillatus Michelsen, 1907. Turiye Parazitol. Derg., v.33, p.254-258, 2009.

BLAGBURN, B. Internal parasites of dogs and cats. Auburm: Novartis, 2010. Available in: <https://www.midamericaagresearch.net/docume nts/Internal\%20Parasite\%20Manual\%20for\%20d ogs.pdf>. Accessed in: 06 Jan. 2019.

EISENBERG, J.F.; REDFORD, K.H. Mammals of the neotropics. Chicago: University Chicago Press, 1999. v.3, p.90-112.

FIELD, S.G.; MICHIELS, N K. Acephaline gregarine parasites (Monocystis sp.) are not transmitted sexually among their lumbricid earthworm hosts. J. Parasitol., v.92, p.292-297, 2006. 
MENEZES, C.C.F. A importância do médico veterinário na saúde pública. 2005. 54f. Monografia (Graduação em Medicina Veterinária) - Universidade Estadual do Ceará, Fortaleza, CE.

MIRANDA, F. Cingulata (tatus) e pilosa (preguiças e tamanduás). In: CUBAS, Z.S.; SILVA， J.C.R. CATÃO-DIAS， J.L. (Eds.). Tratado de animais selvagens: medicina veterinária. 2.ed. São Paulo: Roca, 2014. p.789806.

SHEATHER, A.L. The detection of intestinal protozoa and mange parasites by a floatation technique. J. Comp. Pathol. Therap., v.36, p.266-275, 1923.

SILVA，K.F.M.; COSTA，J.F.; ANACLETO, T.C.S.; TIMO, T.P.C. Avaliação do risco de extinção de Dasypus novemcinctus Linnaeus,
1758 no Brasil. Inst. Chico Mendes BIO, 2015. Disponível em: <http://www.icmbio.gov.br/portal/faunabrasileira /estado-de-conservacao/7106-mamiferos-

dasypus-novemcintus-tatu-galinha>. Acessado em> 11 jul. 2018.

VELAVAN, T.P.; SCHULENBERG, H.; MICHIELS, N.K. Detection of multiple infections by Monocystis strains in a single earthworm host using ribosomal internal transcribed spacer sequence variation. Parasitology, v.137, p.45-51, 2010.

VIZCAINO, S.F.; LOUGHRY, W.J. (Eds.). The biology of the xenartha. Gainesville: University Press of Florida, 2008. 370p.

WILLIS, H.H. A simple levitation method for the detection of hookworm ova. Med. J. Aust., v.2, p.375-376, 1921. 